\title{
Grupos sanguíneos e lúpus eritematoso crônico discoide *
}

\author{
Blood groups and discoid lupus erythematosus
}

\author{
Andréia de Almeida Tamega ${ }^{1}$ \\ Fabíola de Paula Pereira ${ }^{3}$
}

\author{
Lílian Valéria Guerra da Silva Pinto Bezerra ${ }^{2}$ \\ Hélio Amante Miot ${ }^{4}$
}

\begin{abstract}
Resumo: FundAMENTOS: Lesão discoide é a manifestação cutânea mais comum do lúpus eritematoso, e formas cutâneas crônicas apresentam características imunológicas próprias, direcionadas ao polo Th1. Diversas doenças possuem associação com grupos sanguíneos, o que não foi ainda estudado no lúpus discoide. OвJETIVO: Investigar a associação entre tipos sanguíneos ( $\mathrm{ABO}$ e $\mathrm{Rh}$ ) e lúpus eritematoso discoide. MÉTODOS: Estudo prospectivo tipo transversal envolvendo tipagem sanguínea ABO e Rh, inquérito de dados clínicos e dosagem de FAN e C4 de portadores de lúpus discoide sem critérios de doença sistêmica, atendidos em hospital universitário.

Resultados: Foram incluídos no estudo 69 pacientes, sendo $71,0 \%$ do sexo feminino $(p<0,01)$. A idade do surgimento da doença foi de 32,8 \pm 13,1 anos. Formas disseminadas ocorreram em 47,8\%; história de tabagismo foi verificada em 59,4\%; FAN > 1:160, em 31,9\%; e níveis baixos de C4, em 8,7\%. Não houve diferença significativa entre as frequências dos grupos sanguíneos dos pacientes e da população local; entretanto, o grupo A foi associado às formas disseminadas da doença (OR 4,1 e p < 0,05).

CONCLuSÕES: Grupos sanguíneos de pacientes com lúpus discoide apresentam frequência semelhante à da população; porém, formas clínicas disseminadas foram mais prevalentes entre portadores do grupo A.

Palavras-chave: Epidemiologia; Lúpus eritematoso discoide; Sistema do grupo sanguíneo ABO; Sistema do grupo sanguíneo $\mathrm{Rh}-\mathrm{Hr}$
\end{abstract}

\begin{abstract}
BACKGROUnD: Discoid lesion is the commonest cutaneous finding in lupus erythematosus and chronic types have their own immunological features, with Th1 inflammation profile. Although many diseases have association with blood-group systems, this fact was not enlightened in discoid lupus erythematosus.

OвJестіv: To investigate the association between blood groups (ABO and Rh) and discoid lupus erythematosus.

METHODS: A prospective cross-sectional study assessing clinical information, blood group systems (ABO and Rh), FAN and C4 serum levels from discoid lupus patients without characteristics of systemic disease, was carried out at a clinic from a Brazilian university hospital.

RESULTS: Sixty-nine patients were enrolled in the study, $71.0 \%$ were females $(\mathrm{p}<0.01)$, mean age of disease onset was 32.8 \pm 13.1 years, and disseminate form occurred in $47.8 \%$, smoking was related in $59.4 \%$, FAN $>1: 160$ in $31.9 \%$, and low levels of $\mathrm{C} 4$ in $8.7 \%$. There was no significant difference between the frequency of blood groups from discoid lupus patients and local population, however, blood group A was associated to disseminate forms of the disease (OR 4.1 and $\mathrm{p}<0.05)$.

Conclusions: Discoid lupus erythematosus patients with $\mathrm{ABO}$ and Rh blood groups exhibit similar frequencies as in the general population; nevertheless, disseminate clinical forms were more prevalent among group A patients.

Keywords: $\mathrm{ABO}$ blood-group system; Epidemiology; Lupus erythematosus, discoid; Rh-Hr blood-group system
\end{abstract}

\footnotetext{
Recebido em 18.08.2009.

Aprovado pelo Conselho Consultivo e aceito para publicação em 8.12.09

* Trabalho desenvolvido no Departamento de Dermatologia e Radioterapia da Faculdade de Medicina de Botucatu da Universidade Estadual Paulista (FMB-Unesp) - Botucatu (SP), Brasil.

Conflito de interesse: Nenhum / Conflict of interest: None

Suporte financeiro: Nenhum / Financial funding: None

Médica residente do serviço de dermatologia da Faculdade de Medicina de Botucatu da Universidade Estadual Paulista (FMB-Unesp) - Botucatu (SP), Brasil Médica residente do serviço de dermatologia da Faculdade de Medicina de Botucatu da Universidade Estadual Paulista (FMB-Unesp) - Botucatu (SP), Brasil. Médica residente do serviço de dermatologia da Faculdade de Medicina de Botucatu da Universidade Estadual Paulista (FMB-Unesp) - Botucatu (SP), Brasil Professor assistente doutor, Departamento de Dermatologia e Radioterapia da Faculdade de Medicina de Botucatu da Universidade Estadual Paulista (FMB-Unesp) - Botucatu (SP), Brasil.
}

C 2009 by Anais Brasileiros de Dermatologia 


\section{INTRODUÇÃO}

Lúpus eritematoso crônico discoide (LECD) é a manifestação cutânea mais comum do lúpus eritematoso, acomete cerca de $0,3 \%$ dos pacientes dermatológicos e apresenta particularidades epidemiológicas e imunológicas em comparação com formas sistêmicas. Entretanto, até o momento, sua etiologia não é completamente elucidada. $^{1 \cdot 3}$

Caracteriza-se classicamente por pápulas e placas eritematosas, infiltradas, que evoluem com atrofia central, descamação e formação de espículas córneas. ${ }^{2}$

Acomete principalmente áreas fotoexpostas da face, região cervical e membros superiores, mas também couro cabeludo, pavilhão auricular, mucosas e

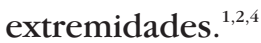

Formas crônicas cutâneas ocorrem, com maior frequência, na faixa entre 20 e 40 anos de idade. Há predomínio de acometimento no sexo feminino, na proporção de 2-3:1, sem predileção por raça. ${ }^{3}$

A classificação do LECD em localizado e disseminado na pele foi originalmente feita por O'Leary em 1934, sendo aceita por autores mais modernos. Considera-se localizada a variedade com lesões restritas ao segmento cefálico e pescoço e disseminada ou generalizada quando, além dessas, existirem lesões no tronco ou membros, independentemente do número. A importância dessa classificação decorre da observação de maior risco de evolução para lúpus eritematoso sistêmico (LES) em pacientes com lesões muito disseminadas na pele. ${ }^{4,5}$

Os exames sorológicos, como a pesquisa de autoanticorpos circulantes, que são tão valorizados no diagnóstico do LES, não são importantes para o diagnóstico do LECD, só sendo utilizados para o diagnóstico diferencial com as formas sistêmicas e para detectar possíveis manifestações do LES. ${ }^{4,6,7}$

Como modelo de doença autoimune cutânea de padrão Th1, o LECD apresenta fundamentos etiológicos multifatoriais de base genética. ${ }^{8}$ Como expressões fenotípicas podem ser estudadas para avaliar padrões de segregação gênica, uma das características que podem ser avaliadas são os grupos sanguíneos.'

Grupos sanguíneos são antígenos de superfície celular expressos segundo padrões de herança bem determinados e estão associados à incidência ou ao prognóstico de diversas doenças, como carcinoma gástrico, tumor pulmonar de pequenas células, carcinoma de esôfago, susceptibilidade a dermatofitoses, líquen plano, dermatite seborreica, lúpus eritematoso sistêmico, câncer de mama, entre outras. ${ }^{9-14}$

Há mais de 30 proteínas e açúcares reconhecidos como marcadores de grupos sanguíneos, entretanto os mais antigênicos compreendem aqueles pertencentes ao sistema $\mathrm{ABO}$ e $\mathrm{Rh}(\mathrm{D})$, segregados independentemente em genes situados nos cromossomos 9q34 e 1 p36. ${ }^{13-16}$
Os autores pretendem estudar a expressão dos grupos sanguíneos (ABO e Rh) em pacientes portadores de LECD.

\section{MATERIAL E MÉTODOS}

Realizou-se estudo prospectivo transversal envolvendo a tipagem sanguínea $\mathrm{ABO}$ e $\mathrm{Rh}$, inquérito de dados clínicos e dosagem de FAN e C4 de portadores de lesões de LECD sem critérios de doença sistêmica, atendidos no ambulatório de dermatologia do Hospital das Clínicas da FMB-Unesp de Botucatu. ${ }^{17}$

Os dados categóricos foram representados como proporções, testados pelo teste do qui-quadrado e análise de resíduos. As associações entre as variáveis foram representadas pelo Odds Ratio (OR). ${ }^{18}$ Posteriormente, as covariáveis foram testadas por um modelo de regressão logística múltipla condicional, usando um algoritmo de redução "stepwise backward" adotando como critérios de entrada e saída das variáveis $\mathrm{p}=0,2$.

Os dados foram tabulados em MS Excel 2003 e analisados com o software SPSS 15.0. ${ }^{19}$ Considerou-se significativo o valor de $\mathrm{p}<0,05$.

\section{RESULTADOS}

Foram incluídos no estudo 69 pacientes, sendo $71,0 \%$ do sexo feminino ( $p<0,01$ ), estabelecendo a razão quanto ao gênero de 2,5:1. As idades dos pacientes e as idades do início da doença estão representadas nos gráficos 1 e 2 , e os principais dados clínicos, epidemiológicos e laboratoriais encontram-se na tabela 1 . Em relação à distribuição topográfica das lesões, observou-se frequência semelhante entre formas localizadas e disseminadas $(p>0,1)$, sendo o segmento cefálico o mais acometido em ambas as formas clínicas.

História de tabagismo durante a doença foi verificada em $59,4 \%$ dos pacientes, porém sem predileção por sexo $(\mathrm{p}>0,1)$.

Títulos positivos de FAN $(>1: 160)$ se associaram à forma disseminada da doença (OR 3,5; IC95\% $1,2$ a 9,$9 ; p=0,01)$.

As frequências dos tipos sanguíneos comparadas com a expectativa regional estão listadas na tabela 2 . Não houve diferença significativa entre a distribuição dos grupos sanguíneos dos pacientes e a da população local $(p>0,1)$, mesmo quando estratificada para grupos $\mathrm{ABO}$ ou Rh. No entanto, o grupo $\mathrm{A}$ foi associado às formas disseminadas da doença (OR 3,1; IC95\% 1,2 a $8,3 ; p<0,05)$. Não se verificou associação entre grupos sanguíneos e sexo, tabagismo, FAN ou C4 $(\mathrm{p}>0,1)$.

Após análise multivariada, o $\mathrm{Rh}$ positivo se associou a início mais tardio da doença (OR 1,3; IC95\% 1,1 a 1,5; p < 0,01), e o grupo sanguíneo A se 


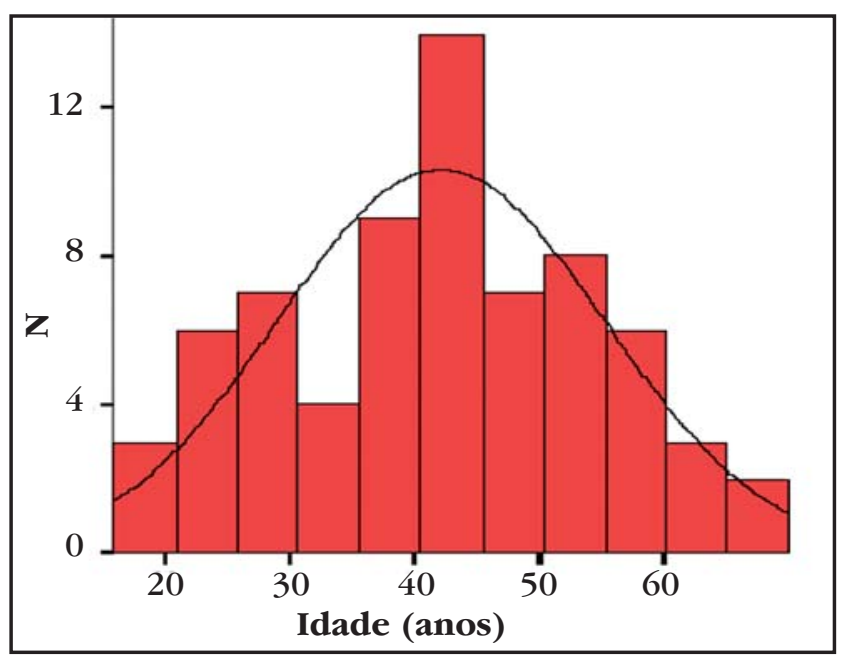

GráfICo 1: Histograma da idade dos pacientes

manteve associado a formas disseminadas (OR 4,1; IC95\% 1,4 a 12,3; p < 0,05).

\section{DISCUSSÃo}

Foram analisados dados epidemiológicos, clínicos e laboratoriais de pacientes portadores de LECD atendidos em serviço universitário no interior do Brasil, sendo os valores encontrados concordantes com a literatura nacional. ${ }^{20-22}$

Investigações de expressão fenotípica em relação a doenças devem ser preferencialmente comparadas com os padrões da população local, pois os aspectos genéticos regionais podem apresentar diferenças entre os povos e, em momentos diferentes da história, numa mesma população. ${ }^{15,21,23}$

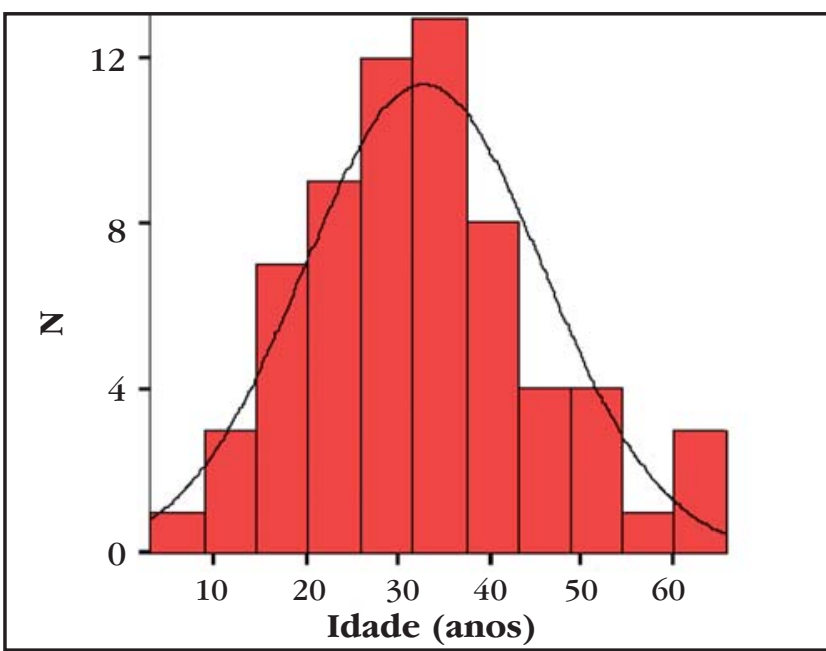

Gráfico 2: Histograma da idade de início da doença

Neste estudo, em que a população estudada possui influência europeia, os tipos sanguíneos mostraram proporções de grupo $\mathrm{Rh}$ positivo e grupo $\mathrm{A}$ mais frequentes que as da literatura do país, porém equivalentes às dos inquéritos entre doadores de bancos de sangue regionais. ${ }^{22,23}$

A hipótese de segregação concomitante dos genes (desequilíbrio genético), ou mesmo da expressão antigênica e produção de anticorpos, pode ser a explicação das associações entre os tipos sanguíneos e diversas doenças. ${ }^{9,11,13,15,24}$

$\mathrm{O}$ antígeno $\mathrm{A}$, identificado neste estudo em associação com doença disseminada, é expresso não somente nas hemácias, mas também na superfície das células nucleadas dos tecidos. Os pacientes do grupo

Tabela 1: Principais dados clínicos, epidemiológicos e laboratoriais dos pacientes com lúpus eritematoso crônico discoide

\begin{tabular}{llll}
\hline Variável & & N & Percentual \\
\hline Sexo & M & 20 & 29 \\
& F & 49 & 71 \\
Forma clínica & Localizada & 36 & 52,2 \\
& Disseminada & 33 & 48,8 \\
História de tabagismo & & 41 & 59,4 \\
FAN > 1:160 & & 22 & 31,9 \\
C4 (níveis reduzidos) & & 6 & 8,7 \\
\hline Idade média (anos) & Média & 42,1 & \\
Idade de início da doença (anos) & Desvio-padrão & 13,2 & \\
& Média & 32,8 & \\
\hline
\end{tabular}


TABela 2: Frequência dos grupos sanguíneos observada entre pacientes com lúpus eritematoso crônico discoide e percentual de valores esperado para a população local

\begin{tabular}{|c|c|c|c|c|}
\hline Grupo ABO & $\mathbf{R h}$ & Lúpus Discoide - N. & $\%$ & Expectativa regional - \% \\
\hline \multirow[t]{3}{*}{ A } & & 36 & 52,2 & 39,7 \\
\hline & Positivo & 32 & 46,4 & 34,9 \\
\hline & Negativo & 4 & 5,8 & 4,8 \\
\hline \multirow[t]{3}{*}{ B } & & 4 & 5,8 & 10,4 \\
\hline & Positivo & 4 & 5,8 & 9,4 \\
\hline & Negativo & 0 & - & 1,0 \\
\hline \multirow[t]{3}{*}{$\mathrm{AB}$} & & 3 & 4,3 & 3,6 \\
\hline & Positivo & 2 & 2,9 & 3,4 \\
\hline & Negativo & 1 & 1,4 & 0,2 \\
\hline \multirow[t]{3}{*}{$\mathrm{O}$} & & 26 & 37,7 & 46,3 \\
\hline & Positivo & 23 & 33,3 & 40,3 \\
\hline & Negativo & 3 & 4,3 & 6,0 \\
\hline \multirow[t]{2}{*}{ TOTAL } & Positivo & 61 & 88,4 & 88,1 \\
\hline & Negativo & 8 & 11,6 & 11,9 \\
\hline
\end{tabular}

A produzem ainda anticorpos (isoaglutininas) contra $\mathrm{O}$ antígeno $\mathrm{B}$. Também as isoaglutininas produzidas como expressões proteicas de membrana celular podem justificar padrões fenotípicos que se relacionem às formas mais graves do lúpus discoide. ${ }^{24}$

Estudos posteriores devem ser conduzidos para avaliar características do lúpus discoide com relação a outros genes do cromossomo 9q34 adjacentes ao lócus do grupo A, assim como homologias do oligossacáride A com antígenos nucleares reconhecidos no lúpus eritematoso.

Formas disseminadas são descritas como relacionadas à maior prevalência de autoanticorpos, maior resistência terapêutica e maior risco de manifestação sistêmica, configurando pior prognóstico. ${ }^{4-6,21,25}$

Ainda de acordo com a literatura, níveis baixos de C4 se correlacionam com a gravidade do lúpus eritematoso e com a frequência de tabagismo, elementos não confirmados com a amostragem deste estudo..$^{21,26}$

Finalmente, grupos Rh positivos foram associados ao surgimento mais tardio da doença, indicando, indiretamente, um fator de maior resistência ao desenvolvimento de lúpus. A grande predominância de grupos Rh positivos pode ser decorrente da descendência europeia que caracteriza a região. ${ }^{22}$ Entretanto, esse aspecto necessita ser cuidadosamente avaliado frente a maiores amostras e ajustado para outras variáveis de confusão, já que, por exemplo, aspectos sócio-econômicos se relacionam a características étnicas e profissionais no país e, indiretamente, ao risco de exposição solar crônica prematura.

\section{CONCLUSÃO}

Grupos sanguíneos de pacientes com LECD apresentam frequência semelhante à da população local; porém, formas clínicas disseminadas foram mais prevalentes entre portadores do grupo A, sugerindo um possível indicador prognóstico da doença.

Novos estudos devem ser conduzidos para avaliar a frequência dos grupos sanguíneos em diferentes populações, avaliando outras variáveis, assim como em outros subtipos de lúpus eritematoso cutâneo. 


\section{REFERÊNCIAS}

1. Werth VP. Clinical manifestations of cutaneous lupus erythematosus. Autoimmun Rev. 2005;4:296-302.

2. Pramatarov KD. Chronic cutaneous lupus erythematosusclinical spectrum. Clin Dermatol. 2004;22:113-20.

3. Prystowsky SD, Herndon JH Jr, Gilliam JN. Chronic cutaneous lupus erythematosus (DLE)--a clinical and laboratory investigation of 80 patients. Medicine (Baltimore) 1976;55:183-91.

4. Patel P, Werth V. Cutaneous lupus erythematosus: a review. Dermatol Clin 2002;20:373-85.

5. Prystowsky SD, Gilliam JN. Discoid lupus erythematosus as part of a larger disease spectrum. Correlation of clinical features with laboratory findings in lupus erythematosus. Arch Dermatol. 1975;111:1448-52.

6. Prystowsky SD, Gilliam JN. Antinuclear antibody studies in chronic cutaneous discoid lupus erythematosus. Arch Dermatol. 1977;113:183-6.

7. Millard LG, Rowell NR. Abnormal laboratory test results and their relationship to prognosis in discoid lupus erythematosus. A long-term follow-up study of 92 patients. Arch Dermatol. 1979;115:1055-8.

8. Wenzel J, Wörenkämper E, Freutel S, Henze S, Haller O, Bieber T, et al. Enhanced type I interferon signalling promotes Th1-biased inflammation in cutaneous lupus erythematosus. J Pathol. 2005;205:435-42.

9. Macsween MP, Syme UA. Abo Blood Groups and Skin Diseases. Br J Dermatol 1965;77:30-4.

10. Balajee SA, Menon T, Ranganathan S. ABO blood groups in relation to the infection rate of dermatophytosis. Mycoses. 1996;39:475-8.

11. Holdsworth PJ, Thorogood J, Benson EA, Clayden AD. Blood group as a prognostic indicator in breast cancer. Br Med J (Clin Res Ed). 1985;290:671-3.

12. Lee JS, Ro JY, Sahin AA, Hong WK, Brown BW, Mountain CF, Hittelman WN, et al. Expression of blood-group antigen A-a favorable prognostic factor in non-small-cell lung cancer. N Engl J Med. 1991;324:1084-90.

13. Tursen U, Tiftik EN, Unal S, Gunduz O, Kaya TI, Camdeviren $\mathrm{H}$, et al. Relationship between $\mathrm{ABO}$ blood groups and skin cancers. Dermatol Online J. 2005;11:44.

14. Chakravartti MR. A statistical appraisal on the relationship between non-ABO blood group systems and diseases. Humangenetik. 1967;5:1-27.

15. Kanbay M, Gür G, Arslan H, Yilmaz U, Boyacioglu S. The relationship of $\mathrm{ABO}$ blood group, age, gender, smoking, and Helicobacter pylori infection. Dig Dis Sci. 2005;50:1214-7.

16. Ayatollahi $H$, Rafatpanah $H$, Khayyami ME, Sayyadpour D, Ravarian M, Sadeghian MH, et al. Association between $\mathrm{ABO}$ and rhesus blood group systems among confirmed human T lymphotropic virus type 1-infected patients in northeast iran. AIDS Res Hum Retroviruses. 2008;24:1155-8.

17. Albrecht J, Werth VP. Development of the CLASI as an outcome instrument for cutaneous lupus erythematosus. Dermatol Ther. 2007;20:93-101.

18. Miot HA, Medeiros LM, Siqueira CRS, Cardoso LC, Gumieiro JH, Pandini Filho MA, et al. Associação entre doença arterial coronariana e as pregas lobular diagonal e anterotragal em homens. An Bras Dermatol. 2006;81:29-33.

19. Mushquash C, O'Connor BP. SPSS and SAS programs for generalizability theory analyses. Behav Res Methods. 2006;38:542-7.

20. Freitas THP, Proença NG. Chronic cutaneous Lupus erythematosus: study of 290 patients. An Bras Dermatol. 2003;78:703-12.

21. Miot HA, Bartoli Miot LD, Haddad GR. Association between discoid lupus erythematosus and cigarette smoking. Dermatology. 2005;211:118-22.

22. Bortolozzi J, de Lucca EJ. [Distribution of ABO and Rh blood groups in Botucatu (Sao Paulo)]. Rev Paul Med. 1971;78:173-6.

23. Curi PR, Kroll LB. [Determination of the sample size and distribution of the $\mathrm{ABO}$ and $\mathrm{Rh}$ blood groups in Botucatu, São Paulo, Brazil (author's transl)]. Rev Bras Pesqui Med Biol. 1976;9:129-35.

24. Ottensooser F, Leon N, de Almeida TV. ABO blood groups and isoagglutinins in systemic lupus erythematosus. Rev Bras Pesqui Med Biol. 1975;8:421-5.

25. Lipsker D, Boeckler P, Hauptmann G. Cigarette smoking and lupus erythematosus. Dermatology. 2006;212:262.

26. Boeckler P, Milea M, Meyer A, Uring-Lambert B, Heid E, Hauptmann G, et al. The combination of complement deficiency and cigarette smoking as risk factor for cutaneous lupus erythematosus in men; a focus on combined $\mathrm{C} 2 / \mathrm{C} 4$ deficiency. $\mathrm{Br} \mathrm{J}$ Dermatol. 2005; $152: 265-70$.

ENDEREÇO PARA CORRESPONDÊNCIA / MAILING ADDRESS:

Hélio Amante Miot

Departamento de Dermatologia, S/N

Faculdade de Medicina da Unesp

Campus Universitário de Rubião Jr.

18618000 Botucatu SP

Tel./fax: (14) 3882-4922

E-mail: beliomiot@fmb.unesp.br

Como citar este artigo/How to cite this article: Tamega AA, Bezerra LVGSP, Pereira FP, Miot HA. Grupos sanguíneos e lúpus eritematoso crônico discoide. An Bras Dermatol. 2009;84(5):477-81. 